\title{
Knowledge and Practice of Nurses and Associated Factors in Managing Neonatal Pain at Selected Public Hospitals in Addis Ababa, Ethiopia, 2020
}

\section{Gemechis Wari (D) \\ Berhanu Wordofa \\ Wudma Alemu ii \\ Teshome Habte (D)}

Department of Nursing, School Nursing and Midwifery, College of Health Sciences, Addis Ababa University, Addis Ababa, Ethiopia
Correspondence: Teshome Habte Tel +25I 9II436I50

Email teshome.habte@aau.edu.et; teshomeh497@gmail.com
Introduction: Hospitalized neonates experience moderate to severe, acute or chronic pain. Recent study indicates that health care providers especially in developing countries have a knowledge and skills gap related to neonatal pain management.

Objective: The aim of this study was to assess the neonatal ICU nurses' knowledge and practice and factors associated with neonatal pain management at selected public hospital of Addis Ababa, Ethiopia.

Methods: Facility-based cross-sectional study design was employed at four selected public hospitals in Addis Ababa, from April to May 2020. A simple random sampling method was used to recruit study participants using a semi-structured and self-administered questionnaire. The logistic regression model was used to identify the association, and odds ratio was used to test the strength of the associations between outcome and predictor variables.

Results: This study was conducted with 119 nursing staffs working in the neonatal intensive care unit with a $96.6 \%$ response rate. The study reveals that $68.7 \%$ of nurses had adequate knowledge and only $32.2 \%$ of them had good practice of neonatal pain management. There was a significant relationship between nurses' knowledge scores and receiving in-service training on neonatal pain management. Having an infant pain management policy in place, getting training on neonatal pain management and knowledge category were factors significantly associated with practice of nurses in neonatal pain management.

Discussion: According to the results of the current research, the majority $(85.2 \%)$ of participants knew that the vital signs of new-borns can be affected by pain. However, only $60.9 \%$ of nurses considered pain as one of the vital signs in new-borns. This indicates that neonatal pain may not be assessed as frequently as a vital sign. And the finding reveals that nurses had poor practice but had adequate knowledge in managing neonatal pain. The respective hospitals and Ethiopian Ministry of Health should provide gap-filling training on neonatal pain management to the nurses.

Keywords: pain, management, neonatal intensive care unit, knowledge, practice, nurses

\section{Introduction}

\section{Background}

The universal and widely cited medical definition of pain is given by the International Association for the Study of Pain (IASP), and it defines pain as a hostile sensual and receptive experience interrelated through concrete or possible injuries. The application of the word pain is learned by each individual through experience associated to sickness early in life. ${ }^{1}$ However, this definition has been 
challenged for the reason that new-borns cannot verbalize their pain, bearing in mind that nonexistence of verbal announcement does not stop one from feeling pain and requiring treatment. Hence, the measurement of neonatal pain is highly dependent on the observer's judgment, and the indicators in the indicating system must be subjectively observed and determined by others. ${ }^{2}$

The cornerstone of pain management is its assessment and measurement especially in case of neonates who cannot report their pain; the assessment ought to comprise physiological (eg, pulse rate, saturation of oxygen) and behavioral factors (eg, cry, facial expression, body movement), and validated pain scale measurement usage is recommended. ${ }^{3,4}$ There are also multiple validated pain scales utilized by NICUs to assess pain, like Neonatal Infant Pain Scale (NIPS), Neonatal Facial Coding System (NFCS), Neonatal Pain, Agitation, and Sedation Scale (N-PASS), Cry, Required Oxygen, Increased Vital Signs, Expression, Sleeplessness Scale (CRIES) and COMFORT Scale for term neonates; the Premature Infant Pain Profile (PIPP) is a validated pain scoring system for preterm neonates. ${ }^{4,5}$

The use of pain assessment scales provides consistency between nurses and other clinicians, and provides an accurate measure for the presence of pain, stress or discomfort, and it can provide an accurate description of the effect of non-pharmacological and pharmacological management interventions on a neonate's pain. ${ }^{6}$

Pharmacological modalities used to treat pain in neonates include: topical anesthetics lidocaine $2.5 \%$, tetracaine $2.5 \%$ and liposomal lidocaine $4 \%$, acetaminophen, local anesthetics like lidocaine injection, sedative medication like morphine, fentanyl and ketamine. ${ }^{4}$

Combination of pharmacological and nonpharmacological, or combination of non-pharmacological therapies provides synergistic effects. Nonpharmacological pain management includes oral sucrose, breast feeding, kangaroo care, swaddling or facilitated tucking, non-nutritive sucking and positioning. ${ }^{4,6}$

Even if pain is reflected to have protective functions, untreated or ineffectively treated pain has both shortand long-term negative effects on the health of the neonate. Among these are the development of complications such as intracranial hemorrhage, decreased immune response, delayed weight gain, prolonged hospitalization, impaired neonate-parent bonding and the development of psychosomatic conditions such as hyperalgesia and allodynia. ${ }^{7}$

\section{Statement of the Problem}

As the World Health Organization has explained, pain is a significant global health problem. However, regardless of huge investment and research efforts in relation to the issue of pain, the clinical practice is far from the recommended one. Pain is under-notified, poorly documented in medical charts and under-treated in both industrialized and unindustrialized countries. ${ }^{8,9}$

The American Academy of Pediatrics states that there is a great prevalence of knowledge divergences in health care professionals' capability to properly assess and manage pain in neonates. ${ }^{10}$ There are many studies reporting that deficiencies are present in knowledge and practice of assessing and treating pain in neonates. Study conducted in Iran showed that $65 \%$ of neonatal nurses did not use any pain assessment tools and the majority of nurses did not feel that pain was being well managed in their neonatal intensive care unit. ${ }^{11}$

Study indicated that the knowledge, practice and beliefs of nurses have a significant influence on the effectiveness of pain management. ${ }^{12}$ Knowledge deficits may cause nurses to hold resilient beliefs regarding neonatal pain management. A study conducted in South Africa showed a knowledge deficit on neonatal pain management associated with the absence of practical guidelines for neonatal pain management. This knowledge deficit is then associated with a lack of pain management in neonatal wards. The study was inspired from 10 years of clinical practice observing that neonatal pain appeared to be the last priority, receiving little or no attention by neonatal staff. $^{13}$

It is hard to find a relevant study that shows the prevalence of pain and its management among hospitalized neonates in Ethiopia. Although various factors surrounding pain management have been explored, little is known about the burden of unrecognized and under-treated pain to neonates in Ethiopia and in Addis Ababa. To my knowledge only limited studies exist that examine knowledge and practice of nursing staff regarding neonatal pain management and associated factors in neonatal units currently at public hospitals of Addis Ababa. As a result, it is valuable to address this gap. This will establish baseline information about the current knowledge and practice of nursing staff regarding neonatal pain management. Consequently, it is a significant step in the process of improving pain management in neonatal units. This in turn will positively reflect on improving the quality of 
life of new-borns and hopefully decrease hospital stays, which in turn will lead to a decrease in medical costs. Therefore, this study aimed to identify nurses' knowledge and practice and factors associated with neonatal pain management in selected public hospitals in Addis Ababa to be used in evidence-based practice and decision-making process.

\section{Methods and Material}

\section{Study Design, Area and Period}

A facility-based quantitative cross-sectional study design was employed among 119 nursing staffs working in the neonatal intensive care unit of four selected public hospitals in Addis Ababa, the capital city of Ethiopia.

The four hospitals with NICU were randomly selected using a lottery method to be included in this study. The selected hospitals are: Tikur Anbessa Specialized Hospital, St. Paul's Hospital Millennium Medical College, Yekatit 12 Hospital and Gandhi Memorial Hospital; currently the total number of NICU nursing staffs in the four selected public hospitals was 150 . The study was conducted from April to May 2020.

\section{Population}

Nursing staffs working in the neonatal intensive care unit of public hospitals in Addis Ababa were the target population. The study subjects were selected by simple random sampling methods from the selected four public hospitals in Addis Ababa during the study period.

\section{Eligibility}

All nurses who had at least six months of work experience in the selected neonatal intensive care units during the study period were included. Nurses who were on annual leave, sick or maternity leave and nurses who had less than six months of work experience were excluded.

\section{Sample Size Determination}

Sample size was calculated by using a single population proportion formula. The proportion means of nurses' knowledge and practice regarding neonatal pain management were taken from a previous related study conducted in Uganda since there is no related previous study in Ethiopia; they were $61.74 \%$ and $52.08 \%$, respectively. ${ }^{14}$ A $95 \%$ confidence level with 0.05 margin of error was assumed.

$$
\begin{gathered}
\mathrm{n}=\frac{(\mathrm{Z} \alpha / 2)^{2} \mathrm{P}(1-\mathrm{P})}{\mathrm{d}^{2}} \\
\operatorname{ni}(1)=\frac{(1.96) 2 \times 0.52(1-0.52)}{(0.05) 2}=383.545 \gg 384 \\
\operatorname{ni}(2)=\frac{(1.96) 2 \times 0.6174(1-0.6174)}{(0.05) 2}=362.98 \approx 363
\end{gathered}
$$

Therefore, the proportion mean of nurses' practice regarding neonatal pain management $(\mathrm{p}=52.08 \%)$ was applied to a large sample size (384) and was used to calculate the final samples for this study.

Since the total population (nurses working in the neonatal intensive care unit of selected public hospitals) was 150 nurses, which was less than 10,000, the adjustment formula was used: $\mathrm{nf}=\frac{\mathrm{ni}}{1+\frac{\mathrm{ni}}{\mathrm{N}}}$

Where $n f=$ final sample size

ni $=$ initial sample size

$\mathrm{N}=$ total population

$$
\mathrm{nf}=\frac{384}{1+\frac{384}{150}}=107.865 \gg 108
$$

After considering a $10 \%$ non-response rate, the final sample size was 119 nurses.

\section{Sampling Procedure}

The number of study units to be sampled from each hospital was determined using a proportional to site allocation formula from the selected four hospitals: $\frac{\mathrm{nf} \times \mathrm{ni}}{\mathrm{N}}$

Where: ni $=$ number of nurses in each of the selected NICU

$\mathrm{nf}=$ the final sample size

$\mathrm{N}=$ the total number of nurses working in the selected NICU

Tikur Anbessa Specialized Hospital $=119 * 33 / 150=$ $26.18 \approx 26$

Saint Paul's Millennium Medical College $=119 * 60$ / $150=47.6 \approx 48$

Yekatit 12 Medical College Hospital $=119 * 34 / 150=$ $26.9733 \approx 27$

Gandhi Memorial Hospital $=119 * 23 / 150=$ $18.2466 \approx 18$

Simple random sampling technique had been used to select nurses from each hospital. Each study unit in the population present during data collection had been represented by a slip of paper; these were put in a box and had been mixed, and a sample of the required size had been drawn from the box. This was done after obtaining the lists 
of nurses from the mentor of nurses, listed alphabetically by name and giving serial number according to their alphabetical order.

\section{Operational Definition}

- Adequate knowledge: Those nurses who scored equal to or greater than $80 \%$ of the knowledge questions correctly.

- Inadequate knowledge: Those nurses who scored below $80 \%$ on knowledge items.

- Good practice: Nurses who scored equal to or greater than $80 \%$ of practice questions correctly.

- Poor Practice: Those nurses who scored below 80\% for practice-related questions correctly.

- Presence of protocol/guideline: Refers to availability of standard neonatal pain management guideline.

- Presence of analgesic: Availability of analgesic drug used for neonatal pain management like opioids and acetaminophen.

- Presence of standard tool: Availability of neonatal pain assessment tool.

\section{Variables of the Study Dependent (Outcome) Variable}

Knowledge and Practice related to neonatal pain management.

\section{Independent Variables}

- Socio-demographic factors: Age, sex, religion, level of education, work experience.

- Environment: Presence of assessment tool, presence of guideline, presence of analgesics and provision of training.

- Organizational factors: Presence of protocol and guideline, presence of analgesics, presence of standardized tool for neonatal pain assessment.

\section{Data Collection Tools and Procedures Data Collection Instrument}

A self-administered structured questionnaire was used to collect the data. It was adapted from a valid and reliable instrument used in previous research done on a similar topic. ${ }^{15,16}$ As the questionnaire was tested against validity and reliability in Brazil and Uganda, it suits the objectives of this study and is also congruent with the system in Ethiopia. The questionnaire consisted of four parts. The first part contains information regarding nurses' profile such as: age, sex, religion, training, years of experience, professional qualification and consists of 8 questions. The second part concerns nurses' knowledge (20 questions) composed of affirmative sentences by using the Likert scale, and answered as follows: I disagree (1 point), I partially disagree ( 2 points), I do not know (3 points), I agree (4 points) and I strongly agree (5 points). These were graded as 1-5 and transformed, computed and recoded to a 3-point rating scale as disagree (1) to agree (3). The third part concerns nurses' practice (15 questions), with answers as follows: never (1), rarely (2), sometimes (3), most of the time (4) and always (5). These were transformed, computed and recoded to a 3-point rating scale as never (1) to always (3). The fourth part is about institutional factors (six questions) and is answered using yes/no.

\section{Data Collection Procedure}

Data had been collected from $20^{\text {th }}$ April to $20^{\text {th }}$ May 2020, after permission was obtained from the selected hospitals. A total of two BSc nurses working in Addis Ababa health institutions for data collection and one first-year MSc nurse student for supervision were recruited and participated throughout the data collection and were trained for one day by the principal investigator on the objective, confidentiality of information, respondents' rights, informed consent and technique of questionnaire administration. The aim of the study, consent form, confidentiality issue and informed consent had been explained and ensured to all of the study subjects. The supervisor and the principal investigator were closely following the data collection process through the data collection period and check for missed values.

\section{Data Quality Assurance}

A structured, validated and reliable adapted questionnaire was used to collect the data; to ensure further the quality of the data collection tool, a pre-test was done on $5 \%(6$ nurses, working in Ras-Desta hospital) of the total calculated sample size two weeks before the actual data collection, and the questionnaire was checked for its clarity, understandability and simplicity. After pre-test, the questionnaires were reviewed and reformatted based on the inputs and comments generated by seniors. The filled questionnaires were checked for completeness to assure the quality of data. Both the principal investigator and recruited supervisors were responsible for supportive supervision on the spot and for reviewing all filled questionnaires on a daily basis. 


\section{Data Processing and Analysis}

The data were checked for completeness and consistency. Then, the data were coded and entered into Epi data v4.6.0.2 for cleaning and transferred to SPSS version 26 for further analysis. Finally, the data were processed descriptively and analytically. The descriptive analysis was used to process and present the frequencies of some of the constructs, such as socio-demographic attributes, of the knowledge and practice of nurses on neonatal pain management. The knowledge and practice of nurses regarding neonatal pain management were obtained after transforming and scoring all variables related to knowledge and practice. Using and modifying McDonald's criteria measuring learning outcome where knowledge and practice are categorized into five levels as very low $(<60 \%)$, low (60-69.99\%), moderate (70-79.99\%), high $(80-89.99 \%)$ and very high $(90-100 \%),{ }^{17}$ percentage scores were used to categorize knowledge and practice into two as "adequate" and "inadequate" for knowledge and "good" and "poor" for practice, respectively. The analytical method was used to determine the predicting variables of nurses' knowledge and practice on neonatal pain management. The data were, thus, fitted to the logistic regression model. The predictive variables were identified in two stages. First, a bivariate analysis was conducted to identify factors with $p<0.25$ at $95 \%$ CI. Then the identified factors were entered into a multivariate analysis in order to control the confounding factors. Finally, those factors with $p<0.05$ at $95 \%$ CI were considered as the predictors of NICU nurses' knowledge and practice on managing neonatal pain. The findings are presented using text, tables, graphs and figures.

\section{Results}

\section{Socio-Demographic Characteristics of the Participants}

A total of 119 questionnaires were distributed, of which 115 $(96.6 \%)$ were fully filled out and collected back. The majority $(89.6 \%)$ of responders were females; 91 (79.1\%) were aged between 24-28 years with a mean age of $26.7 \pm 2.907$ years, and 61 (53.0\%) were single. Additionally, 93 (80.9\%) were BSc nurses, and 78 (67.9\%) had an NICU experience ranging from six months to two years. Regarding their training in neonatal pain management, $61(53.0 \%)$ reported being taught about it during their BSc study, and 60 (52.2\%) reported receiving in-service training.

\section{Nurses' Knowledge About Neonatal Pain Management}

In order to assess nurses' knowledge regarding neonatal pain management, the participants were asked to answer 20 questions, using a Likert scale of five (disagree, partially disagree, I do not know, agree and strongly agree), which then were transformed to a 3-point rating scale ranging from 1 (disagree) to 3 (agree). The total score ranged from 20 to 60 , and, based on the above operational definition, those nurses who scored equal to or greater than $80 \%$ of knowledge-related questions were said to have adequate knowledge.

Accordingly, 79 (68.70\%) of the study participants had adequate knowledge concerning neonatal pain management, while $36(31.30 \%)$ of them had inadequate knowledge.

Out of the total respondents, $104(90.4 \%)$ agreed that preterm new-borns feel pain. As depicted in Table 1, almost all $(112 ; 97.4 \%)$ of the respondents agreed that fullterm new-borns feel pain, while only 3 (2.6\%) of them did not know. The number of respondents who agreed that the vital signs of neonate can be affected by pain was 98 $(85.2 \%)$, while $6(5.2 \%)$ of participants did not know that pain affects a baby's vital signs. There were also 74 participants $(64.3 \%)$ who reported that they knew that light and noise affect new-borns' reaction to pain.

Only 23 respondents (20\%) knew that new-borns' pain is not recognized by professionals. Almost two-thirds of respondents $(70 ; 60.9 \%)$ knew that pain is considered as one of the vital signs in new-borns. The majority (94; $81.7 \%$ ) of respondents reported that pain assessment in new-borns must be systematized. There were also almost the same numbers of respondents, 96 (83.5\%), who knew that pain assessment should be part of the nursing prescription.

\section{Nurses' Practice in Management of Neonatal Pain}

The respondents were also asked to answer five-point Likert scale questions related to nurses' pain management practice in new-borns.

Based on the category specified in the operational definition, about $78(68 \%)$ of the study participants had poor practice in relation to neonatal pain management practice as depicted in Figure 1. The majority (71; $61.7 \%$ ) of the respondents always assess new-born pain through crying, while $61(52.7 \%)$ assess it through 
Table I Nurses' Knowledge in Managing Neonatal Pain in Public Hospitals in Addis Ababa, Ethiopia, 2020 (N= II5)

\begin{tabular}{|c|c|c|c|}
\hline Variables & $\begin{array}{l}\text { Disagree } \\
N(\%)\end{array}$ & $\begin{array}{l}\text { I Do Not Know } \\
N(\%)\end{array}$ & $\begin{array}{l}\text { Agree } \\
\text { N (\%) }\end{array}$ \\
\hline Preterm new-borns feel pain. & $9(7.9)$ & $2(1.7)$ & $104(90.4)$ \\
\hline Full-term new-borns feel pain. & & $3(2.6)$ & $112(97.4)$ \\
\hline $\begin{array}{l}\text { Pain can affect new-borns' heart rate, respiratory rate, temperature, blood pressure, oxygen } \\
\text { saturation, intracranial pressure. }\end{array}$ & II (9.6) & $6(5.2)$ & $98(85.2)$ \\
\hline Pain can affect new-borns' facial expressions, limb movements and crying. & $3(2.6)$ & $6(5.2)$ & $106(92.2)$ \\
\hline Light and noise may affect new-borns' reactions to pain. & $28(24.4)$ & $13(11.3)$ & $74(64.3)$ \\
\hline New-borns' pain is not recognized by professionals. & $77(67.0)$ & $15(13.0)$ & $23(20.0)$ \\
\hline New-borns' pain is not considered by researchers. & $64(55.7)$ & $33(28.7)$ & $18(15.6)$ \\
\hline New-borns react to pain in a particular way. & $25(21.7)$ & $16(13.9)$ & $74(64.4)$ \\
\hline Pain is considered as one of the vital signs in new-borns. & $32(27.8)$ & $13(11.3)$ & $70(60.9)$ \\
\hline Pain assessment in new-borns must be systematized. & $8(7.0)$ & $13(11.3)$ & $94(81.7)$ \\
\hline Pain assessment should be part of the nursing prescription. & $9(7.8)$ & $10(8.7)$ & $96(83.5)$ \\
\hline New-borns require painkillers due to maturity of the nervous system to feel pain. & $18(15.6)$ & $17(14.8)$ & $80(69.6)$ \\
\hline Neonatal pain can be assessed without the use of scales. & $48(4 I .7)$ & $14(12.2)$ & $53(46.1)$ \\
\hline The use of scales for pain assessment is important to the practice. & $18(15.6)$ & $13(11.3)$ & $84(73.1)$ \\
\hline It is important to record pain on the new-borns' chart. & $13(11.3)$ & $13(11.3)$ & $89(77.4)$ \\
\hline Recording pain assessment is a prerequisite to its control. & $9(7.8)$ & $13(11.3)$ & $93(80.9)$ \\
\hline Nurses have sufficient knowledge to assess pain in new-borns. & $24(20.8)$ & $14(12.2)$ & $77(67.0)$ \\
\hline Pain management in new-borns depends on its assessment. & $21(18.3)$ & $10(8.7)$ & $84(73.0)$ \\
\hline Recording new-borns' pain assessment will result in relief. & $20(17.4)$ & $17(14.8)$ & $78(67.8)$ \\
\hline $\begin{array}{l}\text { New-borns submitted to repeated painful procedures may have harmful effects on their } \\
\text { development. }\end{array}$ & $19(16.5)$ & $17(14.8)$ & $79(68.6)$ \\
\hline
\end{tabular}

facial expression. Almost half $(61 ; 52.7 \%)$ of nurses assess new-born pain through vital signs, but 58 $(50.1 \%)$ do not use vital signs as part of neonatal pain assessment. The majority $(78 ; 67.9 \%)$ of participants reported they did not use any scale to assess neonatal pain, while only $18(15.7 \%)$ of them use a scale. The assessed neonatal pain was scored on medical charts by only $17(14.8 \%)$ respondents, while the majority of them did not record neonatal pain on medical charts.

Concerning neonatal pain management, 56 (48.7\%) of participants always use non-nutritive suckling, and $73(63.5 \%)$ of them always encourage breastfeeding to relieve the pain in new-borns. In addition, 52 (45.2\%) nurses always encourage skin-to-skin contact to relieve the pain in new-borns. However, 19 (16.9\%) never encourage skin-to-skin contact to relieve the pain in new-borns.

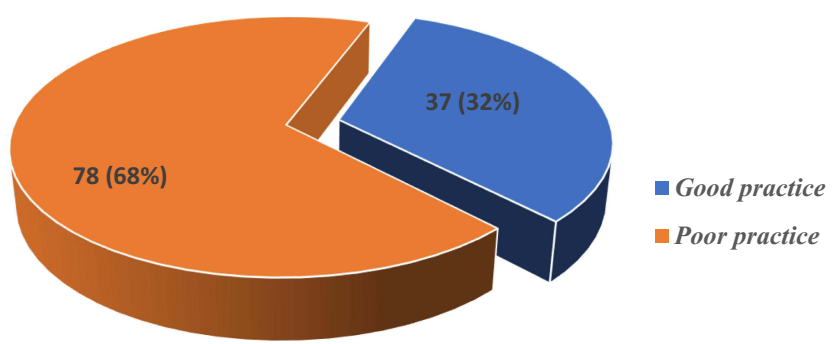

Figure I Category of practice of nurses regarding neonatal pain management in public hospitals in Addis Ababa, Ethiopia, 2020 ( $N=115)$. 


\section{Facility Related Factors Affecting Neonatal Pain Management}

Concerning the question about availability of protocols and guidelines for neonatal pain management in the unit, more than three-fourths $(92 ; 80 \%)$ reported having no guidelines for neonatal pain management. Out of the total participants, $63(54.8 \%)$ reported that analgesics are not available in the unit.

\section{Factors Associated with Knowledge of Nurses on Neonatal Pain Management}

To determine the set of predictor variables which predicted nurses' knowledge of neonatal pain management, bivariate logistic regression was used. Among those predictors, getting training on neonatal pain management while being in NICU, getting training on neonatal pain management at college/university, and nurses' work experiences in NICU were associated with the knowledge of nurses regarding neonatal pain management in bivariate logistic regression analysis $(p<0.25)$. Those variables that have association with the outcome variable in bivariate regression analysis were included in the multiple logistic regression models. Getting training on neonatal pain management while being in NICU and receiving training on neonatal pain management at college/university were significantly associated with the knowledge of nurses regarding neonatal management after controlling for the effect of potentially confounding variables using multiple logistic regression $(p<0.05)$.

Nurses who got training on neonatal pain management while being in NICU were 2.76 times $(\mathrm{AOR}=2.76$; $\mathrm{CI}=$
1.13-6.71) more likely to have adequate knowledge than those who did not get training on neonatal pain management while being in NICU, as shown in Table 2. Those nurses who received training on neonatal pain management at college/university were 2.61 times $(\mathrm{AOR}=2.61$; $\mathrm{CI}=1.04-6.55)$ more knowledgeable than those nurses who did not receive training on neonatal pain management at college/university.

\section{Factors Associated with Practice of Nurses on Neonatal Pain Management}

To determine the set of predictor variables which predicted nurses' practice of neonatal pain management, bivariate logistic regression was used. Among those predictors, gender, marital status, getting training on neonatal pain management while being in NICU, availability of protocol and guidelines for neonatal pain management in the unit, getting training on neonatal pain management at college/ university, knowledge of neonatal pain management and policy on pain management in place were associated with nurses' practice on managing neonatal pain in bivariate logistic regression analysis $(p<0.25)$.

To control the effects of confounding variables, multiple logistic regression models with $p<0.05$ was used, and getting training at college/university, availability of pain management policy in the unit and knowledge of pain management persisted as significant in the multivariate analysis.

Those nurses who had been taught about neonatal pain management at college/university were 2.96-fold $(\mathrm{AOR}=$

Table 2 Factors Associated with Knowledge of Nurses on Neonatal Pain Management in Public Hospitals in Addis Ababa, Ethiopia, $2020(n=115)$

\begin{tabular}{|c|c|c|c|c|c|c|}
\hline \multicolumn{2}{|l|}{ Variables } & \multicolumn{2}{|c|}{ Knowledge } & \multicolumn{2}{|c|}{ Odds Ratio } & \multirow{3}{*}{$\begin{array}{l}P \text {-value } \\
0.042\end{array}$} \\
\hline & & \multirow{2}{*}{$\begin{array}{l}\text { Adequate } \\
35\end{array}$} & \multirow{2}{*}{$\begin{array}{l}\text { Inadequate } \\
8\end{array}$} & \multirow{2}{*}{$\begin{array}{l}\text { OR } \\
(\mathrm{Cl})\end{array}$} & \multirow{2}{*}{$\begin{array}{l}\text { AOR }(\mathrm{Cl}) \\
2.61(1.04-6.55)\end{array}$} & \\
\hline $\begin{array}{l}\text { Receiving training on neonatal pain management at } \\
\text { College/University }\end{array}$ & Yes & & & & & \\
\hline & No & 44 & 28 & I & 1 & \\
\hline \multirow[t]{2}{*}{$\begin{array}{l}\text { Getting training on neonatal pain management being in } \\
\text { NICU }\end{array}$} & Yes & 39 & 9 & $\begin{array}{l}2.93 \\
(1.22- \\
7.00)\end{array}$ & $2.76(1.13-6.71)$ & 0.025 \\
\hline & No & 40 & 27 & I & I & \\
\hline
\end{tabular}

Note: I= Reference category

Abbreviations: OR, odds ratio; AOR, adjusted odds ratio. 
Table 3 Factors Associated with Practice of Nurses on Neonatal Pain Management in in Public Hospitals in Addis Ababa, Ethiopia, $2020(n=115)$

\begin{tabular}{|c|c|c|c|c|c|c|}
\hline \multicolumn{2}{|l|}{ Variables } & \multicolumn{2}{|c|}{ Practice } & \multicolumn{2}{|c|}{ Odds Ratio } & \multirow{3}{*}{$\begin{array}{l}p \text {-value } \\
0.017\end{array}$} \\
\hline & & \multirow{2}{*}{$\frac{\text { Good }}{19}$} & \multirow{2}{*}{$\begin{array}{l}\text { Poor } \\
24\end{array}$} & \multirow{2}{*}{$\begin{array}{l}\text { OR } \\
2.38 \\
(1.06- \\
5.31)\end{array}$} & \multirow{2}{*}{$\begin{array}{l}\text { AOR } \\
2.96(1.21-7.22)\end{array}$} & \\
\hline Receiving neonatal pain management training at University/College & Yes & & & & & \\
\hline & No & 18 & 54 & I & I & \\
\hline \multirow[t]{2}{*}{ There is pain management policy in place } & Yes & 12 & 6 & $\begin{array}{l}5.76 \\
(1.96- \\
16.97)\end{array}$ & $7.87(2.44-25.25)$ & 0.001 \\
\hline & No & 25 & 72 & I & I & \\
\hline \multirow[t]{2}{*}{ Knowledge category } & Adequate & 35 & 44 & $\begin{array}{l}13.5 \\
(3.04- \\
60.22)\end{array}$ & $12.08(2.48-32.78)$ & 0.002 \\
\hline & Inadequate & 2 & 34 & I & I & \\
\hline
\end{tabular}

Note: I = reference category.

Abbreviations: OR, odds ratio; $A O R$, adjusted odds ratio.

2.96; $\mathrm{CI}=1.21-7.22$ ) more likely to have good practice of neonatal pain management than those who did not get training on neonatal pain management at college/university, as depicted in Table 3. Those nurses who had an infant pain management policy in place were 7.87 times $(\mathrm{AOR}=7.87 ; \mathrm{CI}=2.44-25.25)$ more likely to practice good neonatal pain management than those nurses who did not have an infant pain management policy in place. Good practice was 12.08 times $(\mathrm{AOR}=12.08 ; \mathrm{CI}=2.48-32.78)$ more likely among those nurses who had adequate knowledge on neonatal pain management than among those who had inadequate knowledge on neonatal pain management.

\section{Discussion}

\section{Neonatal Intensive Care Unit Nurses' Knowledge on Neonatal Pain Management}

This study revealed that more than half $(68.70 \%)$ of the nurses had adequate knowledge on neonatal pain management. This indicates the majority of nurses were knowledgeable about neonatal pain, and this may be due to their level of education as almost all of them were Bachelor degree holders. In fact, as knowledge increases, so the care for neonatal pain management also increases. This is similar to a study done in Rwanda, which revealed special ranks' knowledge of neonatal pain management. ${ }^{16}$

On the other hand, the current study shows that a few nurses did not know that new-borns feel pain. This may contribute to ineffective pain management in neonates among nurses. However, three-fourths (74.3\%) of the respondents knew that the use of a scale for pain assessment is important to the practice. This indicates that the majority of nurses were aware that the use of a scale in the assessment of neonatal pain results in appropriate intervention. This is higher than in the study conducted in Brazil, which reported that $62.7 \%$ of nurses use a scale to assess pain in neonates. ${ }^{15}$

According to the results of the current research, the majority $(85.2 \%)$ of participants knew that the vital signs of new-borns can be affected by pain. However, only $60.9 \%$ of nurses considered pain as one of the vital signs in new-borns. This indicates that neonatal pain may not be assessed as frequently as a vital sign. In contrast, a study done in Turkey reflects a higher number. ${ }^{18}$ However, the majority $(68.7 \%)$ of nurses knew that new-borns submitted to repeated painful procedures may have harmful effects on their development, and this implies that they do not expose neonates to repeated painful procedures. In contrast, this was lower than the study conducted in Brazil, which found that $88.4 \%$ knew this. ${ }^{19}$ 


\section{Neonatal Intensive Care Unit Nurses' Knowledge Related to Factors on Management of Neonatal Pain}

The current study showed that nurses' knowledge on management of neonatal pain was positively associated with getting training on neonatal pain management while being in NICU. Those nurses who get neonatal pain management training while being in NICU were 2.76 -fold more likely to have adequate knowledge than those who were not getting the training. Similar studies done in Rwanda and Amhara hospitals on neonatal and pediatric nurses revealed that training on pain management was significantly associated with nurses' knowledge of pain management. ${ }^{16,20}$ This suggests that presence of formal training improves the nurses' knowledge to assess and manage pain properly. Consequently, continuous professional training and enhanced at-work focus on standard practice are key to ensure adequate knowledge and good performance.

The current study also indicated that those nurses who studied neonatal pain management at college/university were 2.76 times more likely to have adequate knowledge on neonatal pain management than those who did not get training on neonatal pain management at college/university $(p=0.025)$. A similar study conducted in Amhara region showed lack of training was one factor which negatively associated with nurses' knowledge on pain management. ${ }^{20}$ Apparently getting training concerning pain assessment tools, principles, techniques of pain assessment and management is required in order to provide the ideal care to a neonate in pain.

\section{Neonatal Intensive Care Unit Nurses' Practice on Management of Neonatal Pain} The present study revealed that more than half $(52.2 \%)$ of nurses had good practice in neonatal pain management scoring. This indicates that the majority of nurses practice better on neonatal pain management and it might be due to their educational level and also having adequate knowledge regarding pain management in neonates. A similar result was revealed in a study conducted in Rwanda. ${ }^{14}$

The use of more than one non-pharmacological method to relieve the pain of a new-born was reported as "always" (30.4\%), "sometimes" (38.3\%) and "never" (31.3\%). This implies poor practice of nurses with the combination of more than one non-pharmacological method of managing pain in neonates. This was in contrast with the studies done in Rwanda and Brazil which reported that the majority of participants always use more than one nonpharmacological intervention to relieve pain in newborns. ${ }^{14,15}$ From the current study, the uses of comfort measures give the impression not understandable as an essential role of nurses. It might be result in ineffective neonatal pain management with comfort.

Assessment of neonatal pain is crucial to professional practice and treatment. Different mechanisms of pain control intervention in neonates, including pharmacological and non-pharmacological, as well as minimizing stressful and invasive procedures, should be part of care protocols in all neonatal intensive care units as recommended by the American Association of Pediatrics. ${ }^{10}$ The current study demonstrated that more than half $(57.4 \%)$ of respondents always use pharmacological and non-pharmacological combined to relieve pain in new-borns. This indicates that effective management of pain in neonates was provided. However, a study done in Turkey showed that only $24.5 \%$ of the nurses used pharmacologic and nonpharmacologic methods combined when children experienced pain. ${ }^{21}$

This study indicates that half of the respondents never assess new-borns' pain as a vital sign, implying that the nurses did not consider pain to be a vital sign in newborns, as recognition of pain as the fifth vital sign produces significant changes in professional practice. This indicates the necessity for developing protocols for assessing and treating pain in the neonatal care unit. ${ }^{22}$

\section{Neonatal Intensive Care Unit Nurses' Practice Related to Associated Factors on Management of Neonatal Pain}

This study indicated that availability of pain management policy in the unit, getting neonatal pain management training at college/university and knowledge of pain management significantly associated with the practice on neonatal pain management. The current study indicated that the availability of infant pain management policy was significantly associated with the practice of nurses concerning neonatal pain management.

Those nurses who had an infant pain management policy in place were 7.87 -fold more likely to display good practice in managing neonatal pain than those nurses who did not have a pain management policy in place ( $p=$ $0.001)$. Similarly, a study conducted in Iran on pain management perceptions of the neonatal nurses in NICU and 
neonatal units revealed that the infant pain management policy was associated with practice of nurses in managing infant pain. ${ }^{23}$ This implies that the presence of an infant pain management policy in the unit is a key point in the practice of neonatal pain management and results in effective managing of pain in neonates among nurses. As a result, the presence of an infant pain management policy and management issues was key to following good practice.

This study also revealed that good practice in managing neonatal pain was indicated among those nurses who had received training concerning neonatal pain management at university/college, compared to among those nurses who had not received training on neonatal pain management at university/college. This is similar to a study done in Amhara region. ${ }^{20}$ This implies that receiving neonatal pain management at university/college improves nurses' neonatal pain management practice effectively.

Finally, those respondents who had adequate knowledge were 12.08 -fold more likely to follow good practice on neonatal pain management than those who had inadequate knowledge on neonatal pain management $(p=$ $0.002)$. This denotes that adequate provision of neonatal pain management by nurses can be improved with the knowledge they have regarding neonatal pain management. Those nurses who have adequate knowledge emphatically practice pain management in neonate effectively. This study is consistent with the studies done in Rwanda and Amhara region in Ethiopia which revealed that knowledge of pain management is significantly associated with practice. ${ }^{14,20}$

\section{Limitation of the Study}

- Private hospitals were not included, and the result could not represent them.

- There was limited study in the country to compare the result.

- As this was a cross-sectional study due to time constraint, it did not address the cause-and-effect relationship between the determinant and the outcome of interest.

\section{Conclusion and Recommendation}

\section{Conclusion}

Most nurses had poor practice in managing neonatal pain, but more than half of nurses had adequate knowledge concerning neonatal pain management. However, nurses had inadequate knowledge and poor practice on neonatal pain management related to assessment of neonatal pain without using pain scale and not considering and assessing neonatal pain as part of the vital signs. Furthermore, there was a gap between their knowledge and practice, and we found a significant positive association between the following: getting training on neonatal pain management and knowledge; getting neonatal pain management education at college/university and knowledge; presence of infant pain management policy in the unit and practice; knowledge and practice; and getting neonatal pain management education at college/university and practice.

\section{Recommendations}

The following recommendations are forwarded based on the findings of this study:

\section{To the Respective Hospitals}

- Provide continuous on-job training related to neonatal pain management to all nurses working in NICU.

- It is also helpful to implement an infant pain management policy in the hospitals.

\section{To the Ethiopian Ministry of Health and Nursing Schools}

The Ministry of Health and the Nursing College should review the educational curriculum in order to include neonatal pain management.

\section{To Researchers}

Further research with qualitative study design is also recommended to evaluate more accurately nurses' knowledge and practice as well as attitude and perception on neonatal pain assessment.

\section{To Professional Nurses}

- Nurses need to further improve their knowledge and practice on neonatal pain management.

- Those nurses who had adequate knowledge and good practice on neonatal pain management should also teach their respective colleagues who had deficits in pain management in neonates.

\section{Abbreviations}

BSc, Bachelor of Science; IASP, International Association for the Study of Pain; MSc, Master of Science; NFCS, 
Neonatal Facial Coding System; NICU, neonatal intensive care unit; NIPS, Neonatal Infant Pain Scale; N-PASS, Neonatal Pain, Agitation, and Sedation Scale; SPSS, Statistical Package for Social Sciences.

\section{Ethical Approval and Consent to Participate}

Ethical approval and clearance were obtained from the Institutional Review Board of the Department of Nursing and Midwifery, College of Health Sciences, Addis Ababa University. The objectives of the study were explained to study participants. Verbal and written informed consent were obtained from study participants. All collected information was kept confidential. Coding and aggregate reporting were used in data presentation to ensure anonymity. The study methods were in accordance with the Declaration of Helsinki guidelines and regulations.

\section{Author Contributions}

All authors made a significant contribution to the work reported, whether that is in the conception, study design, execution, acquisition of data, analysis and interpretation, or in all these areas; took part in drafting, revising or critically reviewing the article; gave final approval of the version to be published; have agreed on the journal to which the article has been submitted; and agreed to be accountable for all aspects of the work.

\section{Disclosure}

All authors reported no conflicts of interest for this work. The authors followed all ethical principles of scholarship in the preparation, data collection, analysis, interpretation and completion of this thesis. All scholarly matter addressed in the thesis has been given recognition through citation (cited and referenced) all sources used to prepare this document. This thesis is partial fulfillment of the requirement for graduation as Master's degree from the School of Nursing and midwifery post-graduate Studies at Addis Ababa University. Hard copy of the thesis is in the Library of Addis Ababa University and available to the user under the rules of the library and soft copy found at the university website at: (http://etd.aau.edu.et/bitstream/ handle/123456789/23688/Gemechis\%20Wari.pdf?). We have included acknowledgements and conflict of interests. We did not receive any financial support from our organization and we confirm that this work is original and has not been published elsewhere nor is it currently under consideration for publication by any journal.

\section{References}

1. Cohen M, Quintner J, van Rysewyk S. Reconsidering the International Association for the Study of Pain definition of pain. Pain Rep. 2018;3(2):2. doi:10.1097/PR9.0000000000000634

2. Anand KJ, Craig KD. New perspecives on defination of pain. Pain. 1996;67(1):4.

3. Cong X, McGrath JM, Cusson RM, Zhang D. Pain assessment and measurement in neonates; an updated review. Adv Neonatal Care. 2013;13(6):379-395. doi:10.1097/ANC.0b013e3182a41452

4. Witt N, Coynor S, Edwards C, Bradshaw H. A guide to pain assessment and management in the Neonate. Curr Emerg Hosp Med Rep. 2016;4(1):1-10. doi:10.1007/s40138-016-0089-y

5. Lago PG, Marazi E. Pain Management in the Neonate. Acta Paediatr. Oslo, Norway: Kenya Pediatric Association; 1992:132-139.

6. Perry M, Tan Z, Chen J, Weidig T, Xu W, Cong XS. Neonatal pain: perceptions and current practice. Crit Care Nurs Clin North Am. 2018;30(4):549-561. doi:10.1016/j.cnc.2018.07.013

7. Sharek PJ, Powers R, Koehn A, Anand KJS. Evaluation and development of potentially better practices to improve pain management of neonates. Pediatrics. 2006;118(Supplement 2):S78-S86. doi:10.1542/peds.2006-0913D

8. Linhares MB, Doca FN, Martinez FE, et al. Pediatric pain: prevalence, assessment, and management in a teaching hospital. Braz $J$ Med Biol Res. 2012;45(12):1287-1294. doi:10.1590/S0100879X2012007500147

9. Steven BJ, Abbott LK, Yamada J. Epidemiology and management of painful procedures in children in Canadian hospitals. Can Med Assoc J. 2011;183(7):E403-E410. doi:10.1503/cmaj.101341

10. AAP. Committee on fetus and newborn and section on anesthesiology and pain medicine. Prevention and management of procedural pain in the neonate: an update. Pediatrics. 2016;137(2):e20154271.

11. Mehrnoush N, Ashktorab T, Heidarzadeh M, Momenzadeh S, Khalafi J. Pain management perceptions of the neonatal nurses in NICUs and neonatal units in Ardebil, Iran. Iran J Neonatol. 2016;7 (4):23-29.

12. Sujatha SS, Sundaresan R. Nurses knowledge and neonatal pain management. Int $J$ Adv Res. 2015;3(8):732-737.

13. Khoza SLT, Tjale AA. Knowledge, attitudes and practices of neonatal staff concerning neonatal pain management. Curationis. 2014;37 (2):1-9. doi:10.4102/curationis.v37i2.1246

14. Muteteli C 1, Tengera O 1, Gowan M 1,2. Neonatal pain management among nurses and midwives at two Kigali hospitals Rwanda. $J$ Med Health Sci. 2019;2(2):138-146.

15. Costa T, Rossato LM, Bueno M, et al. Nurses' knowledge and practices regarding pain management in newborns. Rev Esc Enferm USP. 2017;51:1-7. doi:10.1590/s1980-220x2016034403210

16. Lunkuse E . Neonatal pain: nurses' perception of management in a referal hospital in Rwand; 2017.

17. McDonald RP, Ho MH. Principles and practice in reporting structural equation analyses. Psychol Methods. 2002;7(1):64-82. doi:10.1037/ 1082-989X.7.1.64

18. Cırık VA, Çiftçioğlu Ş, Efe E. Knowledge, practice and beliefs of pediatric nurses about pain. J Pediatr Res. 2019;6(3):220-227. doi:10.4274/jpr.galenos.2019.48344

19. Mundim de Oliveira I, Corrêa Castrai T, Peres Cavalcante MM, Calatayud Carvalho J, Firmino Daré M, Marques Salge AK. Nursing professionals' knowledge and attitude related to assessment and treatment of neonatal pain. Rev Eletr Enf. 2016;18:e1160.

20. Mihretu E. Knowledge, attitude, practice, and factors associated with pain management for hospitalized children among nurses; 2018. 
21. Aydin D, Sahiner NC, Ciftci EK. Non-pharmacological strategies used to reduce procedural pain in infants by nurses at family health centres. JPMA. 2017;67(6):889-894.

22. Araujo L, Romero C. Pain: evaluation of the fifth vital sign. A theoretical reflection. Sociedade Brasileira Para o Estudo Da Dor. 2016;16(4):291-296.
23. Mehrnoush N, Ashktorab T, Momenzadeh S, et al. FactorsI nfluencing neonatal pain management from the perspectives of nurses and physicians in a neonatal intensive careunit. Iran J Pediatr. 2017;28 (1):e10015. doi:10.5812/ijp.10015

\section{Publish your work in this journal}

The Journal of Multidisciplinary Healthcare is an international, peerreviewed open-access journal that aims to represent and publish research in healthcare areas delivered by practitioners of different disciplines. This includes studies and reviews conducted by multidisciplinary teams as well as research which evaluates the results or conduct of such teams or healthcare processes in general. The journal covers a very wide range of areas and welcomes submissions from practitioners at all levels, from all over the world. The manuscript management system is completely online and includes a very quick and fair peer-review system. Visit http://www.dovepress.com/testimonials. php to read real quotes from published authors. 